\title{
Magnetic resonance imaging of craniovertebral structures: clinical significance in cervicogenic headaches
}

\author{
Heidi Knackstedt $\cdot$ Jostein Kråkenes • \\ Dalius Bansevicius • Michael Bjørn Russell
}

Received: 5 May 2010/Accepted: 9 September 2011 / Published online: 27 September 2011

(c) The Author(s) 2011. This article is published with open access at Springerlink.com

\begin{abstract}
This paper aims to investigate the relevance of morphological changes in the main stabilizing structures of the craniocervical junction in persons with cervicogenic headache (CEH). A case control study of 46 consecutive persons with CEH, 22 consecutive with headache attributed to whiplash associated headache (WLaH) and 19 consecutive persons with migraine. The criteria of the Cervicogenic Headache International Study Group (CHISG) were used for diagnosing $\mathrm{CEH}$; otherwise the criteria of the International Classification of Headache Disorders (ICHD II) were applied. All participants had a clinical interview, and physical and neurological examination. Proton weighted magnetic resonance imaging (MRI) of the craniovertebral junction, and the alar and transverse ligaments were evaluated and blinded to clinical information. The MRI of the craniovertebral and the cervical junctions, the alar and transverse ligaments disclosed no significant differences between those with CEH, WLaH and or migraine. The site of $\mathrm{CEH}$ pain was not correlated with the site of signal intensity
\end{abstract}

H. Knackstedt $(\bowtie) \cdot$ D. Bansevicius

Department of Neurology, Innlandet Hospital Trust, 2418

Elverum, Norway

e-mail: heidi.knackstedt@sykehuset-innlandet.no

H. Knackstedt · D. Bansevicius · M. B. Russell

Head and Neck Research Group, Research Centre,

Akershus University Hospital, Lørenskog, 1478 Oslo, Norway

J. Kråkenes

Department of Radiology, Section for Neuroradiology,

Haukeland University Hospital, University of Bergen Medical

School, 5021 Bergen, Norway

M. B. Russell

Campus Akershus University Hospital,

University of Oslo, 1474 Nordbyhagen, Norway changes of the alar and transverse ligaments. In fact, very few had moderate or severe signal intensity changes in their ligaments. MRI shows no specific changes of cervical discs or craniovertebral ligaments in CEH.

Keywords Cervicogenic headache - Alar ligaments . Transverse ligaments · Craniovertebral junction . Cervical junction $\cdot$ MRI

\section{Introduction}

Cervicogenic headache (CEH) is a symptomatic headache characterized by chronic unilateral headache possibly secondary to dysfunction of the cervical spine [1-3]. CEH is often worsen by neck movement, sustained awkward head position, external pressure of the upper cervical or occipital region on the symptomatic side [1,2]. Anaesthetic blockades of cervical structures or related nerves can temporarily abolish pain in CEH patients, which may suggest that the pain could be attributed to a neck disorder or structural lesion [1,2,4]. Clinical and/or imaging evidence of neck disorder or lesion can be accepted as a valid cause of headache. However, there is an agreement that degenerative changes in the cervical spine do not necessarily correlate with pain $[1,5]$. Nevertheless, the research is striven to identify causative changes in the cervical spine, which may be attributed to CEH. The craniovertebral junction is stabilized by joint capsules, tectorial membrane, transverse and alar ligaments. Those anatomic structures are innervated by $\mathrm{C} 2$ root [6]. Convergence of the nociceptive afferents of the trigeminal and upper three cervical spinal nerves onto the second-order neurons in the trigemino-cervical nucleus in the upper cervical spinal cord referrers the pain from the cervical spine to the head $[7,8]$. 
The pain in CEH may originate from various anatomic structures in the cervical spine. A German study suggests that lower cervical disc prolapse may cause CEH [9]. It is conceivable that injury to the ligamentous structures can trigger $\mathrm{CEH}$. High-resolution proton density-weighted magnetic resonance imaging (MRI) can visualize structural changes of ligaments and membranes in the upper cervical spine, and it is possible to grade the severity of these structural changes [10-12]. The diagnostic value of such changes is still controversial and their relevance in CEH is unknown. The aim of our study was to examine the frequency of structural changes in the alar and transverse ligaments in persons with CEH, whiplash associated headache $(\mathrm{WLaH})$ and migraine.

\section{Materials and methods}

\section{Study sample}

The case-control study included patients referred to a general neurological outpatient clinic (Dept. of Neurology, Innlandet Hospital, Norway). A total of 118 participants were eligible for the study, but 31 refrained from participation. Of the 87 participants, 46 had CEH, 22 had WLaH, and 19 had migraine. The participants were interviewed and examined by a neurological resident (HK). CEH was classified according to the criteria of the Cervicogenic Headache International Study Group (CHISG) requiring at least three criteria to be fulfilled, not including a Greater Occipital Nerve (GON) blockade, i.e. criterias 1a, 1a1, 1a2, $1 \mathrm{~b}, 1 \mathrm{c}$ and/or III (Table 1), [13]. Otherwise, the criteria of the International Classification of Headache Disorders (ICHD II) were applied [1]. WhipLash was defined by an acceleration/deceleration trauma that caused flexion/ extension distortion of the neck followed by pain/stiffness. Three persons (two with CEH and one with migraine) refrained from MRI due to claustrophobia and two persons with CEH were excluded due to reduced image quality, ending up with 82 participants.

Magnetic resonance imaging protocol and evaluation

We examined the craniovertebral junction in three orthogonal planes (Siemens Symphony, Erlangen, Germany). The persons were scanned in supine position using both the neck coil and the attachable anterior element from the head coil. Images were obtained using a fast spin-echo (SE) T2 and proton-density-weighted sequences.

\section{MR protocol}

We did a T2-weighted series covering the whole cervical spine. Repetition time (TR) and echo time (TE) were TR/ TE 3,360/103, slice thickness $3 \mathrm{~mm}$ without gap, number of excitation (nex) 3, matrix $276 \times 512 \mathrm{~mm}$ and field of view $(\mathrm{FoV}) 280 \times 280 \mathrm{~mm}$. We did proton-weighted series of the craniovertebral junction with $1.5 \mathrm{~mm}$ slice thickness without gap covering the alar and the transverse ligaments in three orthogonal planes. Axial series (12 images) covered from the base of the dens upward, TR/TE 2,660/15, matrix $276 \times 512$, nex 5, FoV $200 \times 165 \mathrm{~mm}$. Coronal series (13 images) covered from anterior atlantal arch backward, TR/TE 2,870/15, matrix $271 \times 512$, nex 5, FoV $200 \times 200 \mathrm{~mm}$. Sagittal series (20 images) covering the entire length of both alar ligaments, TR/TE 2,150/15, matrix $211 \times 512$, nex 3 , FoV $200 \times 150 \mathrm{~mm}$.

Table 1 The diagnostic criteria of cervicogenic headache by the Cervicogenic Headache International Study Group

I. Symptoms and signs of neck involvement
Ia. Precipitation of head pain, similar to the usually occurring one
Ia (1) by neck movement and/or sustained, awkward head positioning, and/or
Ia (2) by external pressure over the upper cervical or occipital region on the symptomatic side
Ib. Restriction of the range of motion (ROM) in the neck
Ic. Ipsilateral neck, shoulder or arm pain of a rather vague, non-radicular nature, or-occasionally-arm pain
of a radicular nature
II. Confirmatory evidence by diagnostic anaesthetic blockades
III. Unilaterality of the head pain, without side shift
IV. Moderate-severe, non-throbbing pain, usually starting in the neck. Episodes of varying duration, or
fluctuating, continuous pain
V. Only marginal effect or lack of effect of indometacin. Only marginal effect or lack of effect of ergotamine
and sumatriptan. Female sex. Not infrequent occurrence of head or indirect neck trauma by history, usually
importance
of more than only medium severity
Other features of lesser
importance
VI. Various attack-related phenomena, only occasionally present, and/or moderately expressed when present:
(a) nausea, (b) phono- and photophobia, (c) dizziness, (d) ipsilateral "blurred vision", (e) difficulties
swallowing, (f) ipsilateral oedema, mostly in the periocular area

It is obligatory that one or more of the phenomena Ia-Ic are present 
Table 2 Demographic data

\begin{tabular}{llll}
\hline & $\begin{array}{l}\text { Cervicogenic } \\
\text { headache } N=46\end{array}$ & $\begin{array}{l}\text { Whiplash associated } \\
\text { headache } N=22\end{array}$ & $\begin{array}{l}\text { Migraine } \\
N=19\end{array}$ \\
\hline Women $(n)$ & 36 & 13 & 17 \\
Men $(n)$ & 10 & 9 & 2 \\
Age mean (SD) & $43.2(9.2)$ & $41.5(7.1)$ & $42.3(11.2)$ \\
Age range (year) & $27-61$ & $27-57$ & $21-58$ \\
Age at onset mean years (SD) & $31.3(11.9)$ & $33.4(9.8)$ & $19.9(8.1)$ \\
Headache duration mean (SD) & $12.4(10.4)$ & $8.6(7.1)$ & $22.1(11.6)$ \\
\hline
\end{tabular}

The classification of the alar and transverse ligament lesions is based on the ratio between any high-signal part and the total cross-sectional area of the ligament. The alar and the transverse ligaments were graded according to the following criteria: grade 0 -ligament with low signal throughout the entire cross-section; grade 1-ligaments with high signal in $<1 / 3$ or less of cross-section; grade $2-$ high signal in $1 / 3-2 / 3$ of cross-section and grade 3-high signal in $>2 / 3$ or more of cross-section. Both sides of the alar and transverse ligaments were visualized in all participants $[10-12,14]$.

\section{MRI evaluation}

All MR images were evaluated by an experienced consultant in Neuroradiology (JK), who was blinded to clinical information.

\section{Statistical analysis}

The statistical analysis was performed using SPSS Base System for Windows 15.0 for all four MRI gradings and dichotomized groups (Grade $0-1$ and Grade 2-3). We used the $\chi^{2}$-test with $5 \%$ level of significance.

\section{Ethical issues}

The Regional Committees for Medical Research Ethics and the Norwegian Social Science Data Services approved the project. The participants that received GON blockade were informed about the procedure and side effects. All participation was based on informed consent.

\section{Results}

Table 2 shows demographic data of the participants. Signal intensity changes in the alar and transverse ligaments were found in $43 \%(n=18)$ of persons with CEH, in $41 \%$ $(n=9)$ in persons with WLaH and in 50\% $(N=9)$ of the persons with migraine. The results were dichotomized in two groups between none to mild (grade $0-1$ ) and moderate to severe (grade 2-3) signal intensity changes. Table 3 shows that moderate to severe signal intensity changes in any of the transverse or alar ligaments (graded 2-3) were equally distributed on the right and left side and there were no statistical significant differences between the CEH, WLaH or migraine groups. Only $16 \%$ had moderate or severe signal changes. Mild signal intensity changes (grade 1) were found in 21,32 , and $44 \%$ of the subjects with CEH, WLaH and migraine, respectively. We disclosed no statistical significant changes regarding side of the change or between the CEH, WLaH and migraine groups dichotomizing the groups into none and mild to severe signal intensity changes (graded 0 and 1-3).

Table 4 shows disc degeneration. Moderate or severe degeneration of the craniovertebral and cervical discs was rare and only found in the $\mathrm{C} 4 / 5, \mathrm{C} 5 / 6$ and $\mathrm{C} 6 / 7$. Changes were seen in all three diagnostic groups, although there were no significant differences among the groups.

Signal intensity changes in the transverse and alar ligaments in relation to the location of the CEH are shown in Table 5. The statistical analyses showed no significant correlation between the site of signal intensity change and site of CEH. Dichotomizing the results in none and mild to severe signal intensity changes did not change the outcome of the analyses.

\section{Discussion}

We found no significant difference in MRI signal intensity changes in the alar and transverse ligaments or any difference in disc degenerative between subjects with $\mathrm{CEH}$, $\mathrm{WLaH}$ and migraine. However, the pain in CEH may originate from various other structures in the cervical spine and cervical ligaments not identified with this MRI protocol which focused on certain structures [15]. But still all pathological changes in the cervical spine with sensory connection to the spinal tract of the trigeminal nerve might potentially be the pain generating structures which has to be focused on [7]. The alar ligament system is involved during cervical extension, lateral flexion, and ipsilateral rotation; nevertheless we found no correlation between side 
Table 3 Signal intensity changes in any of the transverse or alar ligaments (details for grading is described in "Materials and methods" section)

n.s. denotes non-significant

Table 4 Signal intensity changes in the craniovertebral and cervical junction (details for grading is described in "Materials and methods" section)

n.s. denotes non-significant

\begin{tabular}{|c|c|c|c|c|}
\hline & $\begin{array}{l}\text { CEH } \\
N=42 \%(n)\end{array}$ & $\begin{array}{l}\text { WLaH } \\
N=22 \%(n)\end{array}$ & $\begin{array}{l}\text { Migraine } \\
N=18 \%(n)\end{array}$ & $p$ value \\
\hline \multicolumn{5}{|c|}{ Right alar ligament } \\
\hline Grade $0-1$ & $86(36)$ & $86(19)$ & $89(16)$ & \multirow[t]{2}{*}{ n.s. } \\
\hline Grade $2-3$ & $14(6)$ & $14(3)$ & $11(2)$ & \\
\hline \multicolumn{5}{|c|}{ Left alar ligament } \\
\hline Grade $0-1$ & $86(36)$ & $95(21)$ & $89(16)$ & \multirow[t]{2}{*}{ n.s. } \\
\hline Grade $2-3$ & $14(6)$ & $5(1)$ & $11(2)$ & \\
\hline \multicolumn{5}{|c|}{ Both sides alar ligament } \\
\hline Grade $0-1$ & $83(35)$ & $86(19)$ & 89 (16) & \multirow[t]{2}{*}{ n.s. } \\
\hline Grade $2-3$ & $17(7)$ & $14(3)$ & $11(2)$ & \\
\hline \multicolumn{5}{|c|}{ Right transverse ligament } \\
\hline Grade $0-1$ & $90(38)$ & $95(21)$ & $100(18)$ & \multirow[t]{2}{*}{ n.s. } \\
\hline Grade $2-3$ & $10(4)$ & $5(1)$ & $0(0)$ & \\
\hline \multicolumn{5}{|c|}{ Left transverse ligament } \\
\hline Grade $0-1$ & $88(37)$ & $95(21)$ & $89(16)$ & \multirow[t]{2}{*}{ n.s. } \\
\hline Grade $2-3$ & $12(5)$ & $5(1)$ & $11(2)$ & \\
\hline \multicolumn{5}{|c|}{ Both sides transverse ligament } \\
\hline Grade $0-1$ & $88(37)$ & $91(20)$ & $89(16)$ & \multirow[t]{2}{*}{ n.s. } \\
\hline Grade $2-3$ & $12(5)$ & $9(2)$ & $11(2)$ & \\
\hline
\end{tabular}

\begin{tabular}{|c|c|c|c|c|}
\hline & $\begin{array}{l}\mathrm{CEH} \\
N=42 \%(n)\end{array}$ & $\begin{array}{l}\text { WLaH } \\
N=22 \%(n)\end{array}$ & $\begin{array}{l}\text { Migraine } \\
N=18 \%(n)\end{array}$ & $\mathrm{p}$ value \\
\hline \multicolumn{5}{|l|}{$C 2 / 3$} \\
\hline Grade $0-1$ & $100(42)$ & $100(22)$ & $100(18)$ & n.s. \\
\hline Grade $2-3$ & $0(0)$ & $0(0)$ & $0(0)$ & \\
\hline \multicolumn{5}{|l|}{$C 3 / 4$} \\
\hline Grade $0-1$ & $100(42)$ & $100(22)$ & $100(18)$ & n.s. \\
\hline Grade $2-3$ & $0(0)$ & $0(0)$ & $0(0)$ & \\
\hline \multicolumn{5}{|l|}{$C 4 / 5$} \\
\hline Grade $0-1$ & $88(37)$ & $91(20)$ & $89(16)$ & n.s. \\
\hline Grade $2-3$ & $12(5)$ & $9(2)$ & $11(2)$ & \\
\hline \multicolumn{5}{|l|}{$C 5 / 6$} \\
\hline Grade $0-1$ & $69(29)$ & $91(20)$ & $83(15)$ & n.s. \\
\hline Grade $2-3$ & $31(13)$ & $9(2)$ & $17(3)$ & \\
\hline \multicolumn{5}{|l|}{ C6/7 } \\
\hline Grade $0-1$ & $95(40)$ & $100(22)$ & $100(18)$ & n.s. \\
\hline Grade $2-3$ & $5(2)$ & $0(0)$ & $0(0)$ & \\
\hline \multicolumn{5}{|l|}{ C7/TH1 } \\
\hline Grade $0-1$ & $100(42)$ & $100(22)$ & $100(18)$ & n.s. \\
\hline Grade $2-3$ & $0(0)$ & $0(0)$ & $0(0)$ & \\
\hline \multicolumn{5}{|c|}{ Change any junctions } \\
\hline Grade $0-1$ & $70(29)$ & $86(19)$ & $84(15)$ & n.s. \\
\hline Grade $2-3$ & $30(13)$ & $14(3)$ & $16(3)$ & \\
\hline
\end{tabular}

location of pathological signal intensity (higher signal intensity) in the ligaments and the side location of the $\mathrm{CEH}$ $[16,17]$. The transverse ligaments are strained at various movements of the head, still high-signal intensity (graded 2-3) in those ligaments was rare in all three diagnostic groups. A cross-sectional study applying conventional 
Table 5 Signal intensity changes in the transverse and alar ligaments in relation to location of the cervicogenic headache $(\mathrm{CEH})$

n.s. denotes non-significant

\begin{tabular}{llll}
\hline & \multicolumn{2}{l}{ Grade of structural changes on MRI } & \\
\cline { 2 - 4 } & $0-1$ & $2-3$ & \\
\hline Right-sided CEH $n=19$ & $N(\%)$ & $N(\%)$ & \\
Right alar ligament & $17(89)$ & $2(11)$ & n.s. \\
Left alar ligament & $17(89)$ & $2(11)$ & \\
Right transverse ligament & $15(80)$ & $4(20)$ & n.s. \\
Left transverse ligament & $14(74)$ & $5(26)$ & \\
Left-sided CEH $n=23$ & & $4(17)$ & n.s. \\
Right alar ligament & $19(83)$ & $4(17)$ & \\
Left alar ligament & $19(83)$ & 0 & n.s. \\
Right transverse ligament & $23(100)$ & 0 & \\
Left transverse ligament & $23(100)$ & & \\
\hline
\end{tabular}

cervical MRI found no significant difference between patients with CEH and control subjects [18]. More specifically designed MRI protocols and evaluation grading scales were introduced focusing on the structural assessment of craniovertebral ligaments and craniovertebral junctions in persons with whiplash associated disorders $[12,14,19,20]$. High grade changes were far more frequently observed in cases with a previous whiplash trauma than in a control group using a high-resolution proton density-weighted MRI in three orthogonal planes [10, 11].

There are at least four case control studies that used similar MRI methodology as our present study-two of those studies suggests injury of craniocervical structures, while two recent studies failed to reproduce those findings. A new improved MRI protocol showing the ligaments and membranes in the craniovertebral junction was developed 10 years ago [14]. Further, they studied and classified structural changes in the alar ligaments in the late stage of whiplash injuries by the use of a new MRI protocol [10]. Almost half of whiplash associated disorder (WAD) subjects had structural changes in the alar ligaments, while no grade 2 or 3 lesion was found in the control group. Authors suggest that whiplash trauma might cause permanent damage to the alar ligaments, shown by high-resolution proton density-weighted MRI but the reliability of this classification had to be improved. A similar study has been performed by the same group focusing on MRI changes of the tectorial and posterior atlanto-occipital membranes [11]. A study on the radiologic spectrum of craniocervical distraction injuries used fat suppressed $\mathrm{T} 2$ weighted images a method that might be more sensitive to demonstrate increased signal intensity in the atlantoaxial and atlantooccipital joints, craniocervical ligaments, prevertebral soft tissue and spinal cord than conventional MRI, however, we used a specific MRI protocol developed with special emphasis on imaging the ligaments $[14,21]$. Those studies triggered lively discussion between neurologists and radiologists and there was a need of similar studies from other groups that could confirm diagnostic value of those MRI techniques. Myran et al. [20] compared subjects with WAD, chronic non-traumatic neck pain and subjects without neck pain or previous neck trauma. Alar ligament changes grade 0 to 3 were seen in all three groups. Areas of high-signal intensity (grade 2-3) were found in at least one alar ligament in $49 \%$ of the patients in the whiplash associated disorder grade I-II group, in 33\% of the chronic neck pain group and in $40 \%$ of the control group. The diagnostic value and the clinical relevance of magnetic resonance detectable areas of high intensity in the alar ligaments are questionable. Another study examined ligaments and membranes in the craniocervical junction with MRI in patients with WAD and compared them with healthy control subjects [22]. High-signal intensity of the alar and transverse ligaments was quite common and was reported at an average of about $50 \%$ both among patients and control subjects. The incidence of abnormalities of the tectorial and posterior atlanto-occipital membranes was low in both groups. No statistically significant difference between control subjects and patients with WAD was revealed for any of the structures assessed.

Our study failed to show differences or specific changes of cervical discs or craniovertebral ligaments in any studied group. However, our primary focus was somehow different compared with other similar studies. CEH is a defined headache syndrome, while WLaH or WAD could be defined by different symptoms and only one thing in common-neck trauma in the past. Unilaterality of symptoms in CEH allowed us to look for MRI changes at corresponding side.

Structural alterations of the alar ligaments and upper articular joints are frequent in asymptomatic patients [19]. Focussing on only one particular structural change in the cervical spine might not be a suitable diagnostic method to detect possible pathological finding in patients with $\mathrm{CEH}$. 
Future investigations might have to focus more on the heterogenic origin of $\mathrm{CEH}$ and alternative operational tests in addition to the MRI.

\section{Conclusion}

Morphological MRI changes in craniovertebral ligaments showed similar frequency in patients with $\mathrm{CEH}$ compared to those with WLaH and/or migraine. According to our data, such changes have no established value for the diagnosis or work up of $\mathrm{CEH}$.

Acknowledgments This study was supported by grants from Innlandet Hospital Trust and Campus Akershus University Hospital, University of Oslo. Innlandet Hospital and Akershus University Hospital kindly provided research facilities.

\section{Conflict of interest None.}

Open Access This article is distributed under the terms of the Creative Commons Attribution License which permits any use, distribution and reproduction in any medium, provided the original author(s) and source are credited.

\section{References}

1. Headache Classification Subcommittee of the International Headache Society (2004) The International Classification of Headache Disorders: 2nd edn. Cephalalgia 24:1-160

2. Sjaastad O, Fredriksen TA, Pfaffenrath V, The Cervicogenic Headache International Study Group (1998) Cervicogenic headache: diagnostic criteria. Headache 38:442-445

3. Sjaastad O, Fredriksen TA (2000) Cervicogenic headache: criteria, classification and epidemiology. Clin Exp Rheumatol 18:3-6

4. Sjaastad O (1994) Cervcicogenic headache. In: Merskey H, Bogduk N (eds) Classification of chronic pain. Description of pain syndromes and definitions of pain terms, 2nd edn. IASP press, Seattle

5. Pollmann W, Keidel M, Pfaffenrath V (1997) Headache and the cervical spine: a critical review. Cephalalgia 17:801-816

6. Bogduk N (1992) The anatomical basis for cervicogenic headache. J Manip Physiol Ther 15:67-70

7. Kerr FW (1972) Central relationships of trigeminal and cervical primary afferents in the spinal cord and medulla. Brain Res 43:561-572
8. Bogduk N (2004) The neck and headaches. Neurol Clin 22:151-171

9. Diener HC, Kaminski M, Stappert G, Stolke D, Schoch B (2007) Lower cervical disc prolapse may cause cervicogenic headache: prospective study in patients undergoing surgery. Cephalalgia 27:1050-1054

10. Kråkenes J, Kaale BR, Moen G, Nordli H, Gilhus NE, Rorvik J (2002) MRI assessment of the alar ligaments in the late stage of whiplash injury - a study of structural abnormalities and observer agreement. Neuroradiology 44:617-624

11. Kråkenes J, Kaale BR, Nordli H, Moen G, Rorvik J, Gilhus NE (2003) MR analysis of the transverse ligament in the late stage of whiplash injury. Acta Radiol 44:637-644

12. Kråkenes J, Kaale BR (2006) Magnetic resonance imaging assessment of craniovertebral ligaments and membranes after whiplash trauma. Spine 31:2820-2826

13. Bovim G, Sand T (1992) Cervicogenic headache, migraine without aura and tension-type headache. Diagnostic blockade of greater occipital and supra-orbital nerves. Pain 51:43-48

14. Kråkenes J, Kaale BR, Rorvik J, Gilhus NE (2001) MRI assessment of normal ligamentous structures in the craniovertebral junction. Neuroradiology 43:1089-1097

15. Sjaastad O, Fredriksen TA, Pfaffenrath V (1990) Cervicogenic headache: diagnostic criteria. Headache 30:725-726

16. Ishii T, Mukai Y, Hosono N, Sakaura H, Nakajima Y, Sato Y, Sugamoto K, Yoshikawa H (2004) Kinematics of the upper cervical spine in rotation: in vivo three-dimensional analysis. Spine 29:E139-E144

17. Ishii T, Mukai Y, Hosono N, Sakaura H, Fujii R, Nakajima Y, Tamura S, Sugamoto K, Yoshikawa H (2004) Kinematics of the subaxial cervical spine in rotation in vivo three-dimensional analysis. Spine 29:2826-2831

18. Coskun O, Ucler S, Karakurum B, Atasoy HT, Yildirim T, Ozkan S, Inan LE (2003) Magnetic resonance imaging of patients with cervicogenic headache. Cephalalgia 23:842-845

19. Pfirrmann CW, Binkert CA, Zanetti M, Boos N, Hodler J (2001) MR morphology of alar ligaments and occipitoatlantoaxial joints: study in 50 asymptomatic subjects. Radiology 218:133-137

20. Myran R, Kvistad KA, Nygaard OP, Andresen H, Folvik M, Zwart JA (2008) Magnetic resonance imaging assessment of the alar ligaments in whiplash injuries: a case-control study. Spine 33:2012-2016

21. Deliganis AV, Baxter AB, Hanson JA, Fisher DJ, Cohen WA, Wilson AJ, Mann FA (2000) Radiologic spectrum of craniocervical distraction injuries. Radiographics 20:S237-S250

22. Dullerud R, Gjertsen O, Server A (2010) Magnetic resonance imaging of ligaments and membranes in the craniocervical junction in whiplash-associated injury and in healthy control subjects. Acta Radiol 51:207-212 